\title{
Effect of axial conduction on heat transfer in a rectangular microchannel with local heat flux
}

\author{
Fawen YU*, Tao WANG** and Chengbin ZHANG* \\ * Key Laboratory of Energy Thermal Conversion and Control of Ministry of Education, \\ School of Energy and Environment, Southeast University, Nanjing, Jiangsu 210096, PR China. \\ ** Shanghai Institute of Satellite Engineering, Shanghai 200240, PR China. \\ E-mail: cbzhang@seu.edu.cn
}

Received: 24 October 2017; Revised: 16 March 2018; Accepted: 11 April 2018

\begin{abstract}
Based on the continuum assumption and Navier-Stokes equation, three-dimensional fluid flow and conjugate heat transfer in heat sinks with rectangle microchannels have been studied by numerical simulation. The validation of this approach has been demonstrated by comparisons with analytical solutions. Results were obtained for the detailed description of the local and average heat transfer characteristics including wall and fluid temperatures, heat flux, Nusselt number $(\mathrm{Nu})$. Effects of the Peclet number $(\mathrm{Pe})$, the relative conductivity $\left(k_{w} / k_{f}\right)$ and the relative thickness $(W / D)$ of solid substrate were presented and discussed. At low $P e$ flow, axial conduction dominates the heat transfer, which results in a large upstream region which is pre-warmed by axial conduction, so axial conduction cannot be neglected at small $P e$. The increase of $P e$ induces an enhancement of convection, the heat exchange mainly happens on heat region by convection under this condition. A more uniform streamwise temperature distribution at fluid-solid interface indicates the enhancement of the pre-warmed effect along with the increase of $k_{w} / k_{f}$. The study also reveals that the $N u_{x}$ of low $k_{w} / k_{f}$ material is higher than the $N u_{x}$ of high $k_{w} / k_{f}$ material. The increase of wall thickness has an effect on axial distribution of the $N u_{x}$, the trend is similar to that of an increase of the wall thermal conductivity $k_{w} / k_{f}$. It was concluded that the average Nusselt number is proportional to thermal conductivity of the wall. For low $P e$, the fluid is heated by the wall by means of convection on heat region, when $P e \leq 1$, a slight increase of the $\overline{N u}$ on heat region is shown as the increase of $P e$. When $P e>10$, the convection is the dominant factor in heat transfer, an obvious increase of $\overline{N u}$ is shown with increase of $P e$. For high $P e$ flow, the heat transfer enhancement as increase of $P e$ is weak.
\end{abstract}

Keywords : Microchannel, Laminar flow, Local heat flux, Axial conduction, Heat transfer

\section{Nomenclature}

$a \quad$ thermal diffusivity, $\mathrm{m}^{2} . \mathrm{s}$

$A_{\text {wall }} \quad$ cross-sectional area of the microchannel, $\mathrm{m}^{2}$

D hydraulic diameter, $\mathrm{m}$

$h \quad$ convective heat transfer coefficient of the liquid flow, $\mathrm{W} /\left(\mathrm{m}^{2}{ }^{\circ} \mathrm{C}\right)$

$H \quad$ height of the microchannel heat sink, m, see Fig. 1

$H_{\mathrm{ch}} \quad$ height of the microchannel, m, see Fig. 1

$H_{\mathrm{w} 1} \quad$ substrate thickness on top side of microchannel heat sink, m, see Fig. 1

$H_{\mathrm{w} 2} \quad$ substrate thickness on down side of microchannel heat sink, m, see Fig. 1

$k \quad$ thermal conductivity, $\mathrm{W} /\left(\mathrm{m} .{ }^{\circ} \mathrm{C}\right)$

$L \quad$ length of the microchannel heat sink, m, see Fig. 1 


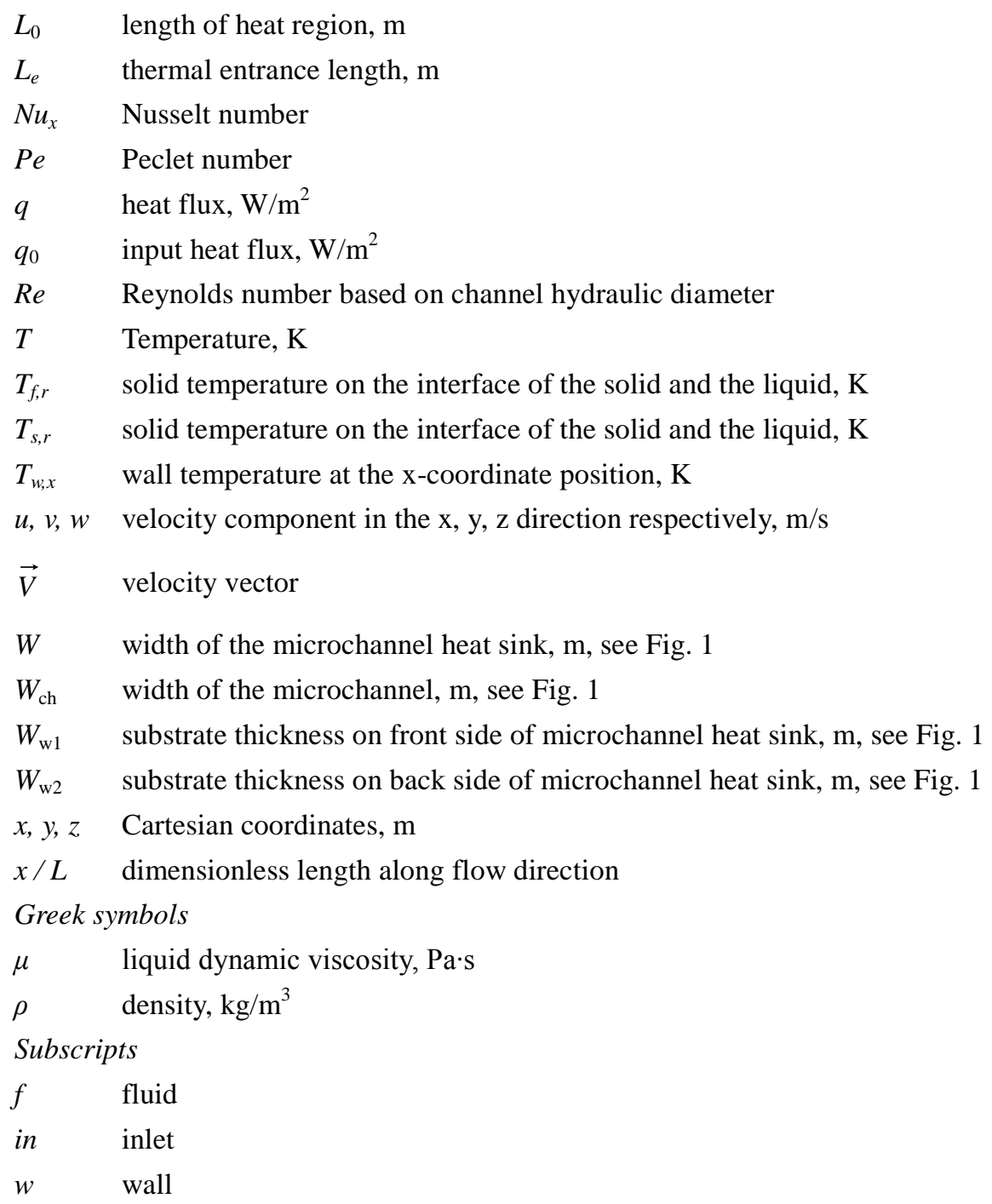

\section{Introduction}

Microchannel heat sink, first introduced by Tuckerman and Pease (1981), has received much attention in recent years due to the high heat transfer efficiency (Wang et al. (2009), Zhang et al. (2010), Ates et al. (2010), Rahimi and Mehryar (2012), Chen et al. (2009)). Along with high development of electronic systems, the continuous increase in heat dissipation at components, module and system levels (the peak local heat flux of these electronic equipments may reach $10^{6} \sim 10^{7} \mathrm{~W} / \mathrm{m}^{2}$ ) leads to an increasing demand for highly efficient electronic cooling technologies (Liu and Chen (2013, 2014), Chen et al. (2012)), so the traditional ways of air cooling technologies cannot meet the requirements of heat dissipation. Among the available heat transfer techniques for electronic cooling including heat pipe (Liu et al. (2013), Deng et al. (2017)), phase change materials (Ashraf et al. (2017)) et al., the microchannel heat sink has been proven to be a high performance cooling method (Mudawar (2001)).

In conventional channels, the axial heat conduction of the solid wall can be neglected in the convection heat transfer due to the fact that the thickness of the solid is usually very small as compared with the hydraulic diameter. On the contrary, the thickness of the solid wall is usually equal in size or lager than the hydraulic diameter of microchannel. Cole and Cetin (2011) studied the wall effects on heat transfer using a layer description of the fluid flow in microchannel, although Cole obtained detailed results for both temperature and Nusselt number for a variety of channel geometries and over a wide range of $P e$ using the infinite-domain Fourier transform, the study just considered the conditions of 2D microchannel, and the heat transfer along circumferential was ignored. Kosar (2010) studied the effects of thermal conductivity and substrate thickness on convective heat transfer in laminar internal flows. The effects of axial heat conduction are more notable under conditions of short channel, thick wall or low Reynolds number 
(Maranzana et al (2004), Hetsroni et al. (2005), Li et al. (2007)). From the heat transport viewpoint, the characteristic time for convection and conduction become comparable for the microscale flow, therefore the convection term no longer dominates the heat transfer in longitudinal direction. In general, the ratio of convection term and diffusion term is characterized by the dimensionless number, Peclet number $(P e)$. The effect of axial conduction in fluid on the heat transfer has been studied for both parallel-plate microchannel (Jeong and Jeong (2006)) and microtube (Cetin et al. (2009)) with boundary conditions defined by constant wall temperature and constant wall heat-flux. In these studies, it can be concluded that the effect of the axial conduction in fluid cannot be neglected when the $P e$ is small.

The local temperature and the local wall heat flux at the interface between fluid and solid which needed in estimating the convection heat transfer coefficients $(h)$ are very difficult to implement during the experiment. Shown by Herwig and Hausner (2003), uniform wall heat flux density along the whole channel and the fluid temperature keep constant during the flow in channel are not always valid for mini-micro-channels. Uneven heating or local heating often appears in microscale heat transfer research. Based on field coordination theory (Guo et al. (1998), WANG et al. (2004)), the intensity of convective heat transfer depends on the coordination relation between the velocity field and the temperature gradient field. Axial heat transfer effect caused by local heating is different from the case which heat source applied to the whole channel. All in all, the interest on the effect of axial conduction on laminar flow and heat transfer in microchannel has received much attention for many years, but the mechanisms of laminar flow and heat transfer in three dimensional microchannel with asymmetric heated by local heat source have not been fully revealed. In our study, the axial conduction in the wall is being taken into account.

In this paper, the study focus on the effect of axial conduction of the wall and the fluid on heat transfer in a three dimensional rectangular microchannel with up wall heated by a local heat source, which has not been studied before. The effect of the axial and circumferential conduction in the wall, the axial conduction in fluid, and the convection between the wall and the fluid are considered conjugately. Results were generated for the detailed description of the local and average heat transfer characteristics including temperatures of wall and fluid, heat flux and $\mathrm{Nu}$. Influences of $P e$, the relative conductivity $\left(k_{w} / k_{f}\right)$ and the relative thickness $(W / D)$ of solid substrate were presented and discussed.

\section{Laminar flow and heat transfer in microchannel}

\subsection{Mathematical Model}

For microchannel liquid flow with the equivalent hydraulic diameter of $1 \mu \mathrm{m} \sim 1 \mathrm{~mm}$, the velocity slip condition can be neglected, and the continuum assumption and the Navier-Stokes equations are applicable (Guo and Li (2003)). Based on the continuum assumption and Navier-Stokes equation, three-dimensional fluid flow and conjugate heat transfer in heat sinks with rectangle microchannels have been analyzed numerically in this paper.

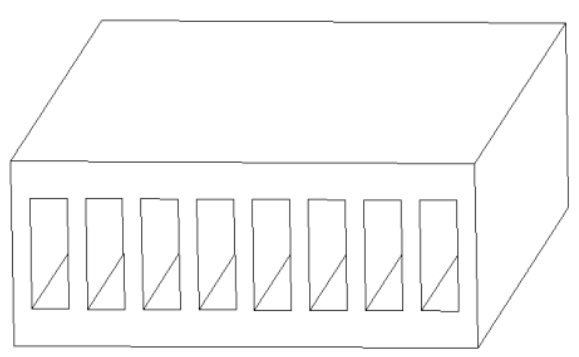

(a)

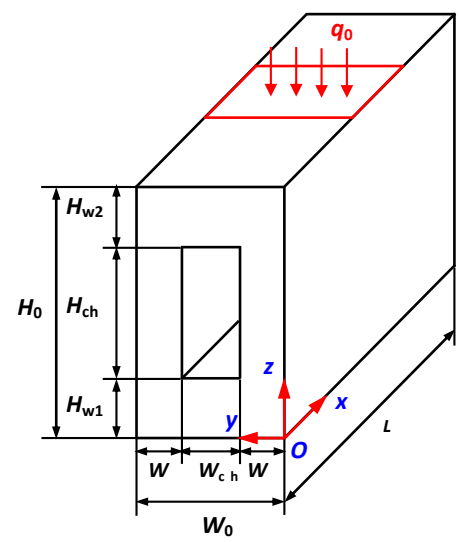

(b)

Fig. 1 Schematic of rectangular microchannel heat sink: (a) structure of parallel microchannel, (b) unit cell of microchannel.

Table 1 Dimensions of microchannel heat sink used in validation (Shah and London (1978))

\begin{tabular}{c|c|c|c|c}
\hline \hline$W(\mu \mathrm{m})$ & $W_{\mathrm{ch}}(\mu \mathrm{m})$ & $H_{\mathrm{w} 1}(\mu \mathrm{m})$ & $H_{\mathrm{ch}}(\mu \mathrm{m})$ & $H_{\mathrm{w} 2}(\mu \mathrm{m})$ \\
\hline 21.5 & 57 & 270 & 180 & 450 \\
\hline
\end{tabular}


The rectangular microchannel heat sink is shown in Fig. 1, where a unit cell consisting of one channel is selected because of the symmetry of the structure. The unit cell can be represented by an asymmetric rectangular channel with the cross-section dimensions as shown in Table 1.

Some assumptions are applied to simplify the model of conventional Navier-Stokes and energy equations. The fluid employed in this study is incompressible, the solid and fluid have constant properties. And the flow is steady laminar flow, and the inner wall is no-slip. In this study, the radiation heat transfer is neglected. Based on the simplified assumptions above, the continuity, momentum and energy equations for a fully developed 3D flow heat transfer are,

$$
\begin{gathered}
\nabla \cdot \vec{V}=0 \\
\rho(\vec{V} \cdot \nabla \vec{V})=-\nabla P+\mu \nabla^{2} \vec{V} \\
\vec{V} \cdot \nabla T=a \nabla^{2} T
\end{gathered}
$$

The energy equation of the solid thermal conduction is,

$$
\nabla^{2} \cdot T=0
$$

Where $P$ is the pressure of liquid, $\rho$ is the density of liquid, $T$ is the temperature of liquid, and $\mu$ is the viscosity, $\vec{V}$ is the velocity vector and $a$ is the thermal diffusion coefficient.

The boundary conditions on the interface between the solid and liquid can be written as

$$
\begin{gathered}
T_{s, r}=T_{f, r} \\
-\left.k_{s} \frac{\partial T_{s}}{\partial n}\right|_{r}=-\left.k_{f} \frac{\partial T_{f}}{\partial n}\right|_{r} \\
\left.u\right|_{r}=\left.v\right|_{r}=\left.w\right|_{r}=0
\end{gathered}
$$

The boundary conductions for velocity and temperature at channel inlet are,

$$
\text { for } x=0, \quad u=u_{\text {in }}, \quad v=w=0, T=T_{\text {in }}
$$

The flow is fully developed at channel outlet,

$$
\text { for } \quad x=L, \quad \frac{\partial u}{\partial x}=\frac{\partial v}{\partial x}=\frac{\partial w}{\partial x}=0, \frac{\partial T}{\partial x}=0
$$

For outer surfaces of the channel, the adiabatic boundary conditions are applied except the heat sink top wall.

$$
\text { for } 1 \leq x / L \leq 2, \quad 0 \leq y \leq W, \mathrm{z}=H, \quad-k_{s} \frac{\partial T}{\partial z}=q_{0}
$$

The local Nusselt number, $N u_{x}$, is used to describe the internal laminar flow and heat transfer characteristics of microflow. $N u_{x}$ along the channel at the $x$-coordinate is,

$$
N u_{x}=\frac{h_{x} L_{0}}{k_{f}}
$$

Where $\overline{T_{w}}=\frac{1}{A_{\text {wall }}} \oint_{\text {wall }} T_{w_{x}}(x, y, z) d S, \overline{T_{f}}=\frac{\int T \rho \vec{v} \cdot d \vec{A}}{\int \rho \vec{v} \cdot d \vec{A}}, \quad h_{x}=\frac{\bar{q}_{x}}{\left(\bar{T}_{w}-\bar{T}_{f}\right)}$.

The average Nusselt number downstream the thermal entrance section is,

$$
\overline{N u}=\frac{\int_{L_{e}}^{L} N u_{x} d x}{L-L_{e}}
$$

Where $L_{e}$ is the length of thermal entrance section.

\subsection{Numerical Method}


The finite-control-volume method and the SIMPLE algorithm together with a uniform structured mesh based on rectangle grid elements were used to obtain the numerical solution of the above model along with the boundary conditions described above. The resulting system of algebraic equations is solved using the Gauss-Seidel iterative technique, with successive over-relaxation to improve the convergence time. Several different mesh sizes are used to test the grid independence. The simulation results obtained using the final mesh system is proved independent of the mesh size. Double precision was used in the computations, and solutions were considered converged when the residual levels of velocity and temperature below $10^{-6}$ and the heat and mass reach balances.

In order to verify the validity of the numerical analysis using the numerical code, the flow and heat transfer characteristics of rectangular microchannel heat sink (the geometric parameters are given in Table 1) are studied. The dimensionless velocity distribution for fully developed flow in microchannel obtained in this section are plotted in Fig. 2. The dimensionless velocity here is defined as $u / u_{\max }$ (in which $u_{\max }$ is the maximum velocity located at the center of rectangular channel). Excellent agreement is found between the present numerical prediction and the analytical solution (Qu and Mudawar (2002), Shah and London (1978)). As can be seen from the comparison between the analytical solutions and numerical velocity profiles at plane $z=0$ and plan $y=0$ which were presented in Fig. 2, the difference is so small that we have reason to believe that the two results are virtually identical.
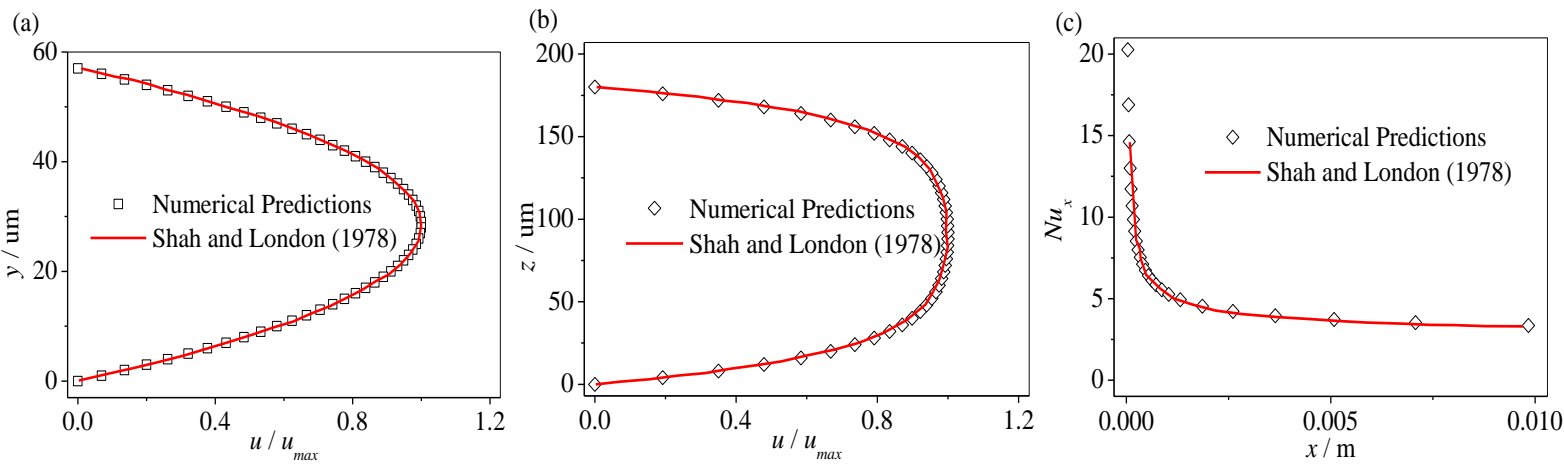

Fig. 2 Comparison between the analytical solutions by Shah and London (1978) and numerical predictions:

(a) dimensionless velocity distribution of $z=0$ section,

(b) dimensionless velocity distribution of $y=0$ section,

(c) $N u_{x}$ distribution along flow direction in a rectangular microchannel.

In order to verify the solution for the energy equation, the surrounding solid region is removed and a pure convective heat transfer problem in the rectangular channel is considered. The constant heat flux applied to the top wall of the channel is uniform. Based on numerical results, the Nusselt number $N u_{x}$ along the channel is evaluated and plotted in Fig. 2(c). Dates in Fig. 2 suggests that the flow characteristics and heat transfer predictions using this mathematical model is accurate for micro-scale flow.

\section{Results and discussion}

Based on the continuum assumption and Navier-Stokes equation, three-dimensional fluid flow and conjugate heat transfer in heat sinks with rectangle microchannels heated over region $1<x / L_{0}<2$ have been analyzed numerically. The liquid in microchannel is deionized water. Dimensions of microchannel heat sink are summarized in Table 2. At first, the effects of the $P e$ number on heat transfer are discussed. Influences of the relative conductivity $\left(k_{w} / k_{f}\right)$ on heat transfer are analysed following. Finally, the variations of the heat transfer characteristics caused by the relative thickness of solid substrate $(W / D)$ are presented and discussed.

Table 2 Dimensions of microchannel heat sink in numerical analysis.

\begin{tabular}{c|c|c|c|c}
\hline \hline$W(\mu \mathrm{m})$ & $W_{\mathrm{ch}}(\mu \mathrm{m})$ & $H_{\mathrm{w} 1}(\mu \mathrm{m})$ & $H_{\mathrm{ch}}(\mu \mathrm{m})$ & $H_{\mathrm{w} 2}(\mu \mathrm{m})$ \\
\hline 100 & 100 & 100 & 100 & 100 \\
\hline
\end{tabular}

$N u_{x}$ is one way to view heat transfer behavior in microchannel. The normalized temperature difference between the wall and the fluid is another perspective to analyze the heat transfer details. The normalized temperature difference 
between the wall and the fluid is,

$$
\left(\overline{T_{w}}-\overline{T_{f}}\right) \cdot k_{f} /\left(q_{0} L_{0}\right)
$$

Where $\overline{T_{w}}=\frac{1}{A_{\text {wall }}} \oint_{\text {wall }} T_{w_{x}}(x, y, z) d S, \overline{T_{f}}=\frac{\int T \rho \vec{v} \cdot d \vec{A}}{\int \rho \vec{v} \cdot d \vec{A}}$.

\subsection{Heat Transfer Behaviours of the Microchannel}

\subsubsection{Effect of Axial Conduction in Fluid}

Peclet number $(\mathrm{Pe})$ describes the ratio of convection and conduction in fluid. The results no considering the wall thermal conduction $(W / D=0)$ for several $P$ e are presented in Fig. 3. The proportion of the convection in heat transfer is proportional to $P e$. But when $W / D=0$, the convective heat transfer only occur in heating region $\left(x / L_{0}=1 \sim 2\right.$, see Fig. 3(a)), so the upstream fluid is heated by heat source through conductivity of the fluid.

With the decrease of $P e$, both the conduction of the fluid and the preheating effect of heat source for upstream fluid increase, so dimensionless average temperature difference between the wall and the fluid $\left(T_{w}-T_{f}\right) * k /\left(q_{0} * L\right)$ increases. Under high $\mathrm{Pe}$ condition, the weak conductivity of the fluid leads to the most heat comes from heat source added to the fluid of heat region, so the maximum of $N u_{x}$ appears at staring of the heat region. And with development of the flow, $N u_{x}$ of heat region decreases (see Fig. 3(c)), this trend is similar to the thermal entrance effect of microchannel. Differently, owing to the strong preheating effect of heat source for upstream fluid for small $P e$, the average temperature difference between the wall and the fluid is large, so the thermal entrance effect of $N u_{x}$ is less obvious at starting of the heat region. And under heat source with constant heat density, the $N u_{x}$ of heat region increases monotonically for small $P e$ condition.

The upstream fluid is heated by the heat comes from the fluid of heat region. The smaller the $P e$ is, the stronger the conduction of the fluid is, and the bigger the temperature difference between the wall and the fluid in upstream fluid is. Therefore, the axial conductivity of the fluid can not be neglected under low Pe condition.
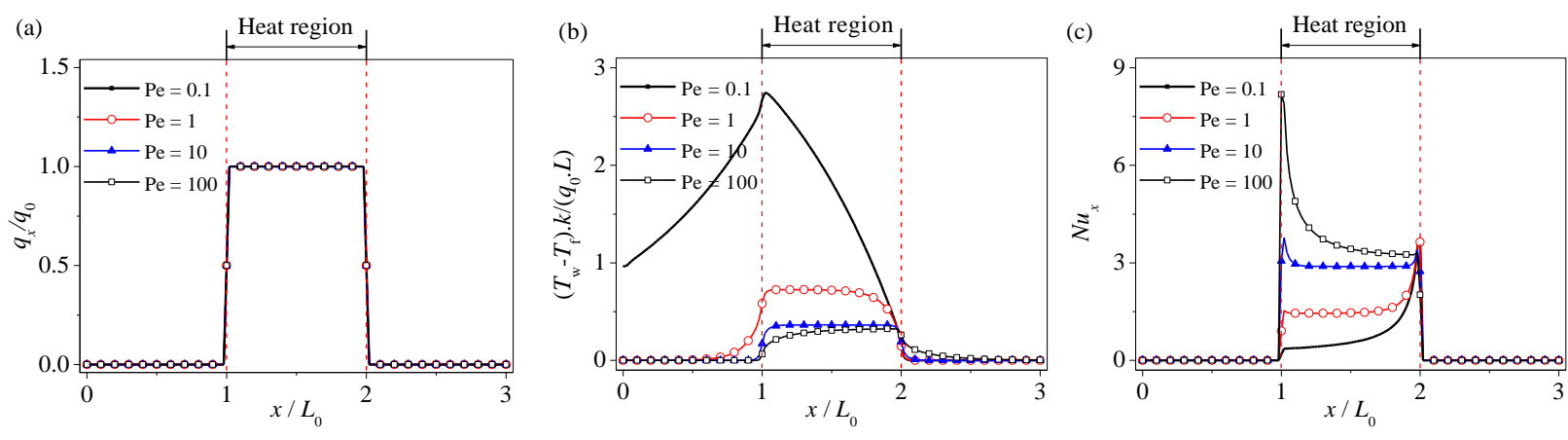

Fig. 3 Effects of $P e$ on (a) $q_{x} / q_{0}$, (b) $\left(T_{w}-T_{f}\right) * k_{f} /\left(q_{0} * L_{0}\right)$ and (c) $N u_{x}$ for $W / D=0$.

\subsubsection{Effect of Axial Conduction in Wall}

Axial conduction of the wall can not be ignored when the hydraulic diameter of the microchannel is close to the thickness of the wall in microscale flow. To analyze the proportion of heat conduction in wall during heat transfer process, Fig. 4 illustrates the flow field distribution under conditions of $P e=0.1 、 k_{w} / k_{f}=3 、 W / D=0,1$.

Different from the case of $W / D=0$, considering the wall heat conduction, a portion heat of heat source transfers to upstream by conduction of the wall, so the thermal entrance effect forms near entrance of the channel, meanwhile the maximum of $q_{x} / q_{0},\left(T_{w}-T_{f}\right)^{*} k /\left(q_{0} * L\right)$ and the $N u_{x}$ appear at this location. Under influences of convective heating of the wall and conduction of the fluid, $\left(T_{w}-T_{f}\right) * k /\left(q_{0} * L\right)$ decreases along flow direction from channel entrance to the end of heat region.

The fluid of the heat region is heated by convection of heat source, Fig. 4(c) also shows that the $N u_{x}$ of heat region increases monotonously. And affected by the convection of the wall, $\left(T_{w}-T_{f}\right) * k /\left(q_{0} * L\right)$ becomes smaller and another peak of $N u_{x}$ appears near the end of heat region. After the entrance, the values mentioned above $\left(q_{x} / q_{0},\left(T_{w}-T_{f}\right) * k /\right.$ $\left(q_{0} * L\right)$ and $\left.N u_{x}\right)$ decrease rapidly. Take no account of the wall conduction $(W / D=0)$, the heat of heat region transfers to upstream merely by conduction of the fluid, so the effect of thermal entrance section does not appears. From the analysis above, we can conclude that the conduction in the wall can not be neglected under low $P e$ conditions. 

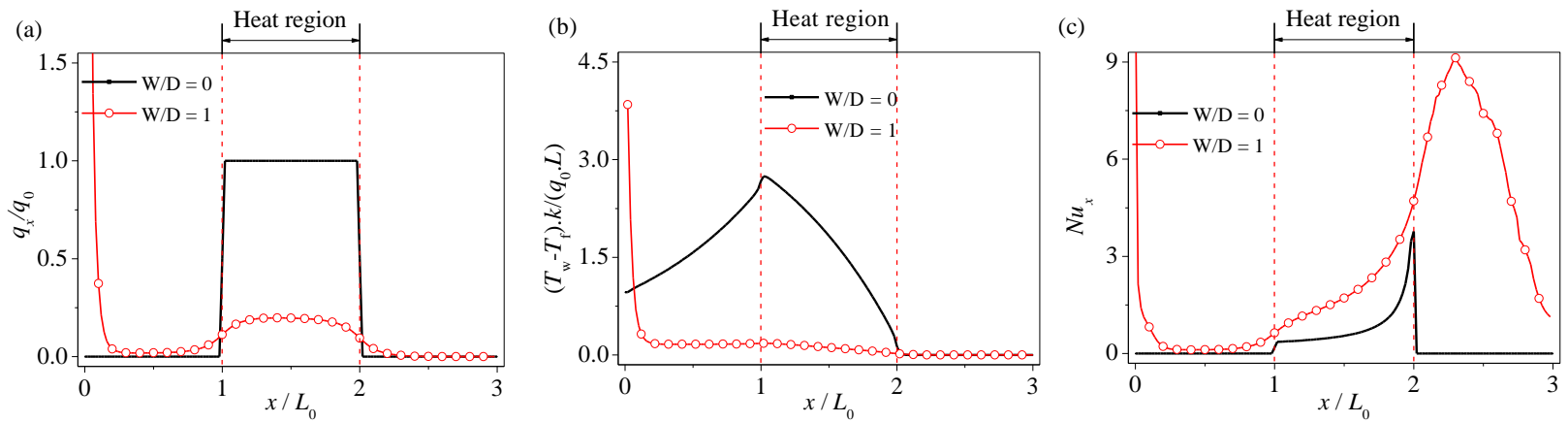

Fig. 4 Effects of $W / D$ on (a) $q_{x} / q_{0}$, (b) $\left(T_{w}-T_{f}\right) * k_{f} /\left(q_{0} * L_{0}\right)$ and (c) $N u_{x}$ for $P e=0.1, k_{w} / k_{f}=3$.

\subsubsection{Effect of the Heat Source Location}

To have an insight into the effects of heat source location, Fig. 5 demonstrates the flow field distributions of $P e=0.1$ 、 $k_{w} / k_{f}=3 、 W / D=1$ under condition of local heating and overall heating. The conduction is the dominant mechanism for heat transfer in the fluid for small $P e$. When heat source added to the whole channel, there is an obvious thermal entrance section near the channel entrance. And the maximum of $q_{x} / q_{0},\left(T_{w}-T_{f}\right) * k /\left(q_{0} * L\right)$ and $N u_{x}$ appear at the channel entrance. After the thermal entrance, $q_{x} / q_{0}$ decreases firstly and then keeps constant almost. Meanwhile the $N u_{x}$ of downstream after thermal entrance decreases rapidly. The fluid is heated by a portion heat of the heat region when the channel heated locally, so the thermal entrance effect forms near channel entrance, and the maximum of $q_{x} / q_{0}$ and $N u_{x}$ appear at channel entrance. At local heating boundary conditions, $q_{x} / q_{0}$ increases again affected by local heat source, in addition $q_{x} / q_{0}$ decrease rapidly in downstream of the heat region (Fig. 5(a)).

The $N u_{x}$ for heat region increases monotonically when the channel heated by whole heat source. Differently, at local heating condition, the $N u_{x}$ increase monotonously near start of the heat region, and the $N u_{x}$ keep increasing until end of the heat region. Affected weakly by heat source, in downstream of the heat region, the $N u_{x}$ decreases again (Fig. 5(c)).

(a)

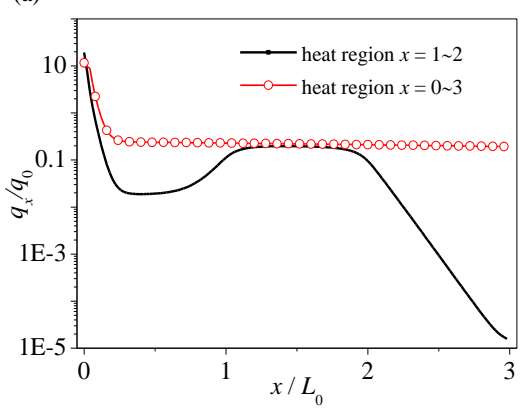

(b)

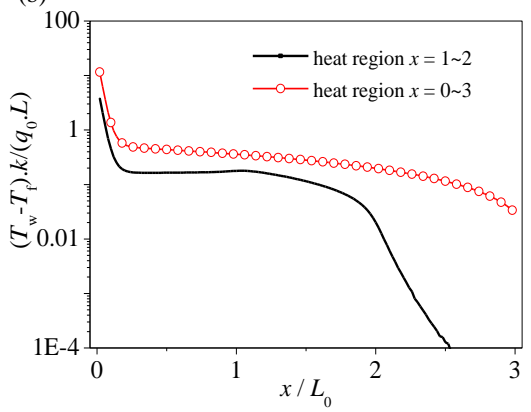

(c)

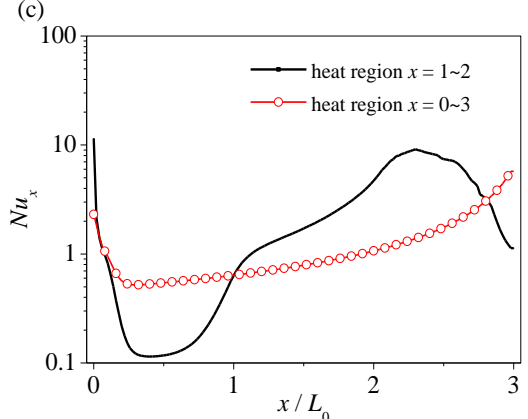

Fig. 5 Effects of the heat source location on (a) $q_{x} / q_{0}$, (b) $\left(T_{w}-T_{f}\right) * k_{f} /\left(q_{0} * L_{0}\right)$ and (c) $N u_{x}$ for $P e=0.1 、 k_{w} / k_{f}=3$.

\subsection{Influences of $P e$}

Dimensionless wall heat flux $q_{x} / q_{0}$ is an important design feature in thermal design. Fig. 6(a) shows $q_{x} / q_{0}$ of different $P e$ as a function of dimensionless longitudinal distance $x / L_{0}\left(L_{0}\right.$ is the length of heat region). Thanks to entrance effect of flow, thin thickness of the boundary layer near channel inlet makes the convective heat transfer resistance smaller, so the coefficient of convective heat transfer enhanced. Attributed to the strong convective heat transfer in developing region of the thermal boundary layer near channel inlet, a high ratio of $q_{x} / q_{0}$ is found near channel inlet. With fast growth of the thermal boundary layer, the convective heat transfer resistance increases, so the convection between the fluid and the wall becomes weaker gradually, and that leads to the decrease of $q_{x} / q_{0}$. As $P e$ increases, the entrance effect on $q_{x} / q_{0}$ decreases, and the length of thermal entrance becomes shorter. The wall conduction makes heat to transfer axially upstream to pre-heat the fluid which has lower temperature than wall. And the effect of axial conduction in fluid becomes more pronounced as $P e$ decreases (Cole and Cetin (2011)). For low $P e$ flow $(P e=0.1)$, the proportion of the convection in heat exchange is small, so the axial conduction along the channel is the main way of heat exchange. At 
$P e=0.1$, the peak of $q_{x} / q_{0}$ appears near channel entrance, it can be observed that the axial conduction in both the fluid and the wall cannot be neglected for small $P e$.

Distributions of $q_{x} / q_{0}$ for different $P e$ which presented in Fig. 6(a) show that $q_{x} / q_{0}$ for high $P e$ is larger than that for low $P e$ in heat region. The increases of $q_{x} / q_{0}$ in heat region also imply the enhancement of convection as $P e$ rises. As can be seen from Fig. 6(a) the heat exchange mainly happens in heat region by convection for high Pe. Data in Fig. 6(b) suggests that the normalized mean temperature difference between the mean temperature of fluid and fluid temperature of inlet $\left(T-T_{\text {inlet }}\right)^{*} k /\left(q_{0} * L\right)$ for each $P e$ tends to rises monotonically along flow direction before end of heat region. The reason is that all the outer boundaries of the channel in addition to the heated region are insulated, so the heat added to fluid remains in fluid. For same fluid properties and channels with fixed geometry, mean velocity of the fluid is proportional to $P e$. With increase of $P e$, the convection between the wall and the fluid becomes more pronounced, and the pre-warmed effect of heat region for upstream becomes weaker, so $\left(T-T_{\text {inlet }}\right) * k /\left(q_{0} * L\right)$ between the wall and the fluid becomes obvious. In Fig. 6(b), at $P e=0.1,\left(T-T_{\text {inlet }}\right) * k /\left(q_{0} * L\right)$ between the wall and the fluid is too small to be visible. Furthermore, $\left(T-T_{\text {inlet }}\right) * k /\left(q_{0} * L\right)$ is fairly close at entrance of heated region $\left(x / L_{0}=1\right)$.

For seek the detailed heat transfer behaviors in microflow, $N u_{x}$ along flow direction is plotted in Fig. 6(c). When $P e$ is small, axial conduction dominates the heat transfer, so a long upstream region is pre-warmed. The evidences are high dimensionless wall heat flux $q_{x} / q_{0}$ near channel entrance as detailed in Fig. 6(a) and small dimensionless temperature difference between the wall and the fluid which displayed in Fig. 6(b) for low $P e$ flow. At $P e=100, N u_{x}$ is large at entrance of heat region $\left(x / L_{0}=1\right)$ and falls monotonously through whole heat region. The distribution of $N u_{x}$ at $P e=10$ shows reasonable behavior as expected. The shape of $N u_{x}$ when $P e=10$ relatively flat than $P e=100$ over the heat region $\left(1<x / L_{0}<2\right)$. Differently, at low $P e$ conditions (such as $P e=0.1,1$ ), the heat transfer between the wall and the fluid is dominated by the axial conduction, the $N u_{x}$ increases monotonically over entire heat region. And it is notable that a local peak of $N u_{x}$ appears in downstream region (about $x / L_{0}=2.25$ ) when $P e=0.1$ and 1 . The local peak of $N u_{x}$ is leaded mainly by two reasons. Under the continuous heating effect of the wall, closely following the end of the heat region (about $x / L_{0}=2.25$ ), the temperature difference between the wall and the fluid is small. And at this location, the ratio of $q_{x} / q_{0}$ is relative high, according to equation (11), a local peak of $N u_{x}$ appears in downstream of the heat region (about $x / L_{0}=2.25$ ).
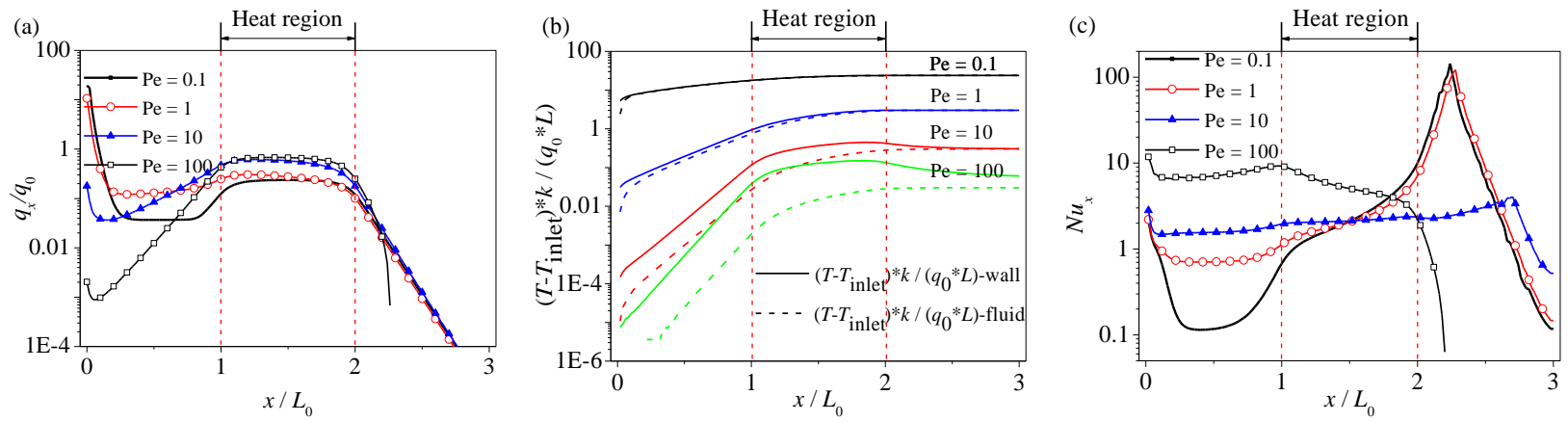

Fig. 6 Effects of $P e$ on (a) $q_{x} / q_{0}$, (b) $\left(T-T_{\text {inlet }}\right) * k_{f} /\left(q_{0} * L_{0}\right)$ and (c) $N u_{x}$ for $W / D=1, k_{w} / k_{f}=3$.

\subsection{Influences of the Wall Thermal Conductivity $\left(k_{w} / k_{f}\right)$}

The effects of axial heat conduction are more relevant for short ducts, thick walls and low Pe. Axial distributions of $N u_{x}$ are plotted for different $k_{w} / k_{f}$ when $W / D=1, P e=100$ (Fig. 7(a)). As can be seen from Fig. 7(a), $q_{x} / q_{0}$ along flow direction becomes more uniform as $k_{w} / k_{f}$ rises. With increase of $k_{w} / k_{f}$, thermal resistance of conduction in wall decreases, so the pre-warmed effects of the heat region in wall is enhanced along with the increase of $k_{w} / k_{f}$. For this reason, a more uniform streamwise temperature distribution at fluid-solid interface can be found for high $k_{w} / k_{f}$. There is reason to believe that the temperature distribution of fluid-solid interface is more uniform in materials with high conductivity than that in ones which have low conductivity.

Moreover, the conductive resistance decreases as $k_{w} / k_{f}$ increases, so $q_{x} / q_{0}$ near channel entrance becomes larger. At $P e=1000$, convection is the dominant mechanism for heat transfer. Judging from the trends of $q_{x} / q_{0}$, it can be obtained that the larger the heat conductivity of the wall is, the wider the area affected by the heat source is. For all cases, the $k_{w} / k_{f}$ of this section is larger than 1, owing to the lower temperature in fluid than in wall, the fluid is heated by the wall. 
Therefore, with increase of $k_{w} / k_{f}$ the heat transfer by conduction of the wall increases, so the distribution of the heat source becomes more uniform, and this contributes to the smaller temperature difference between wall and fluid, that is the local effect of the heat source is less obvious. On the contrary, as $k_{w} / k_{f}$ decreases, the local influence of the heat source becomes more obvious, this trend leads to the temperature difference between wall and fluid increases monotonously. From Fig. 7(b), it can be seen that the temperature difference for $k_{w} / k_{f}=3$ is close to the value which no considering the conduction of the wall.

Affected by the thin boundary layer near channel entrance, the coefficient of convective heat transfer enhanced, a peak of $N u_{x}$ can be found near channel entrance for different $k_{w} / k_{f}$. Owing to the fast growing of thermal boundary layer, the thermal resistance of convective heat transfer increases, $N u_{x}$ decreases quickly closely following the channel inlet because of the weaken convection between fluid and wall. It is obvious in Fig. 7(c), a local peak of $N u_{x}$ can be found near the entrance of heat region $\left(x / L_{0}=1\right)$ for materials with relative low conductivity $\left(k_{w} / k_{f}=3\right)$. The reason is that the enhancement of conduction between wall and fluid near the entrance of heat region. For same $P e$, with increase of $k_{w} / k_{f}$, the influence region of heat source is enlarged by proportion increase of the wall conduction. From Fig. 7(c), one can conclude that the trend of $N u_{x}$ is similar to the trend which heat source added to the whole channel for materials with $k_{w} / k_{f}=300$. Much more, the influence for convection caused by heat source is more pronounced when the wall materials is less conductive relative to the fluid. Namely the $N u_{x}$ of low conductivity material is higher than that for materials with high conductivity. And the conclusion is consistent with results of Nonino et al. (2009).
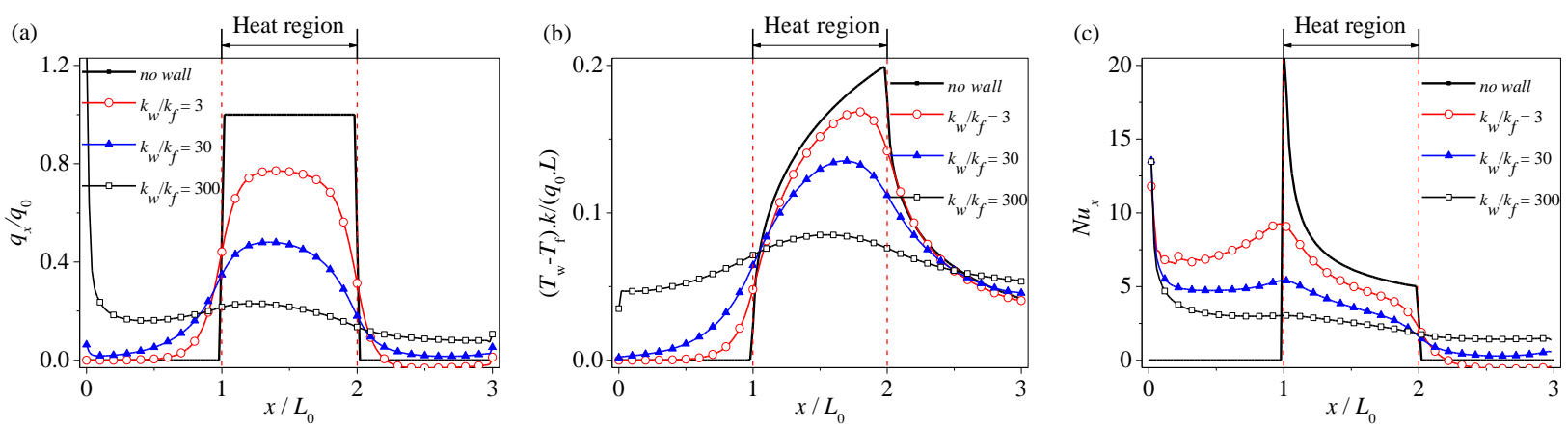

Fig. 7 Effects of $k_{w} / k_{f}$ on (a) $q_{x} / q_{0}$, (b) $\left(T_{w}-T_{f}\right) * k_{f} /\left(q_{0} * L_{0}\right)$ and (c) $N u_{x}$ for $W / D=1, P e=100$.

Figure 8 clearly demonstrates the profiles of $q_{x} / q_{0},\left(T_{w}-T_{f}\right) * k /\left(q_{0} * L\right)$ and $N u_{x}$ for several conductivities when $W / D=$ $1, P e=0.1$. With decreases of $k_{w} / k_{f}$, the heat of wall conduction during heat transfer process decreases, increase of the heat which transfers from heat source to fluid by convection leads to the increasement of $q_{x} / q_{0}$. The proportion of the wall conduction is proportional to $k_{w} / k_{f}$, so with increase of $k_{w} / k_{f}$, the pre-heating effect for fluid by the wall conduction enhances, and this leads to the decreases of $\left(T_{w}-T_{f}\right) * k /\left(q_{0} * L\right)$.

For different $k_{w} / k_{f}$, the trends of $N u_{x}$ are similar. Both in heat region and upstream, the differences of $N u_{x}$ for different $k_{w} / k_{f}$ are small. The reason is that the small proportion of the wall conduction for small $k_{w} / k_{f}$ makes more heat transfers to fluid by convection, by this reason the peak of $N u_{x}$ in downstream region for low $k_{w} / k_{f}$ is bigger than that for high $k_{w} / k_{f}$.
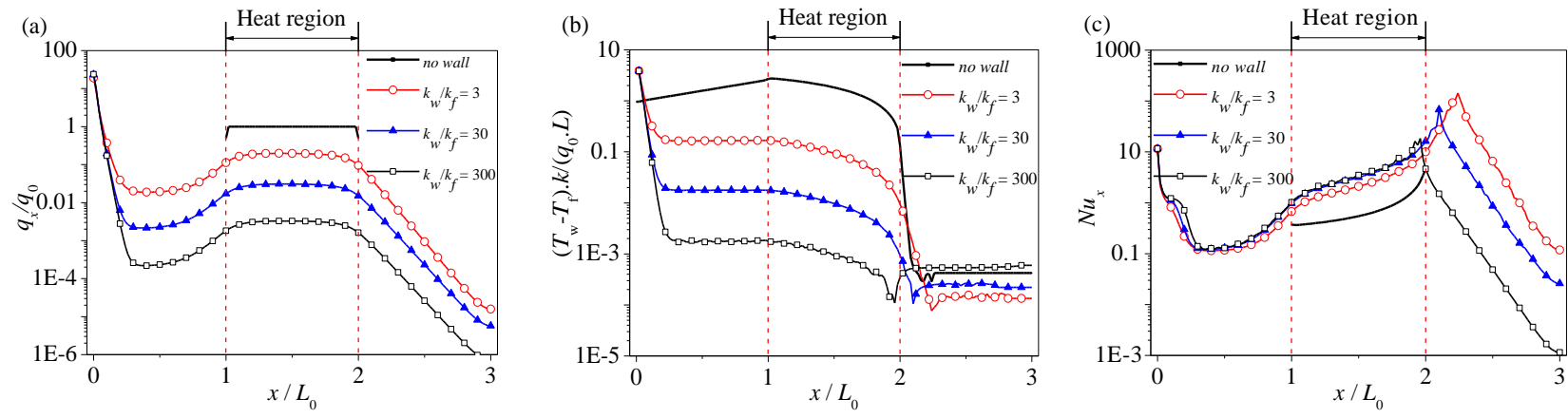

Fig. 8 Effects of $k_{w} / k_{f}$ on (a) $q_{x} / q_{0}$, (b) $\left(T_{w}-T_{f}\right) * k_{f} /\left(q_{0} * L_{0}\right)$ and (c) $N u_{x}$ for $W / D=1, P e=0.1$. 


\subsection{Influences of the Wall Thickness (W/D)}

The distribution of $q_{x} / q_{0},\left(T_{w}-T_{f}\right) * k /\left(q_{0}^{*} L\right)$ and $N u_{x}$ for different $W / D$ when $k_{w} / k_{f}=75 、 P e=1000$ are plotted in Fig. 9. At $k_{w} / k_{f}=75 、 P e=1000$, which is convection dominated, the $q_{x} / q_{0}$ determined by the heat which the wall stored. The increase of $W / D$ enhances the heat transfer rate of the wall, and the enhancement in heat transfer rate of the wall caused by increase of $W / D$ enhances the convection between wall and fluid. And this makes the heat transfer by convection more uniform axially, not only the increase of $q_{x} / q_{0}$ in upstream and downstream, but also the decrease of $q_{x} / q_{0}$ in heat region are the corresponding performances of this trend (see Fig. 9(a)). The local heating effect is weaken by enhancement of the convection owing to the increase of $W / D$. Therefore, with increase of $W / D$ the pre-heating effect of the wall for fluid increases, so $\left(T_{w}-T_{f}\right) * k /\left(q_{0} * L\right)$ decreases. Compared with smaller W/D case, the $\left(T_{w}-T_{f}\right) * k /\left(q_{0} * L\right)$ curve for larger $W / D$ becomes more gentle (Fig. 9(b)).
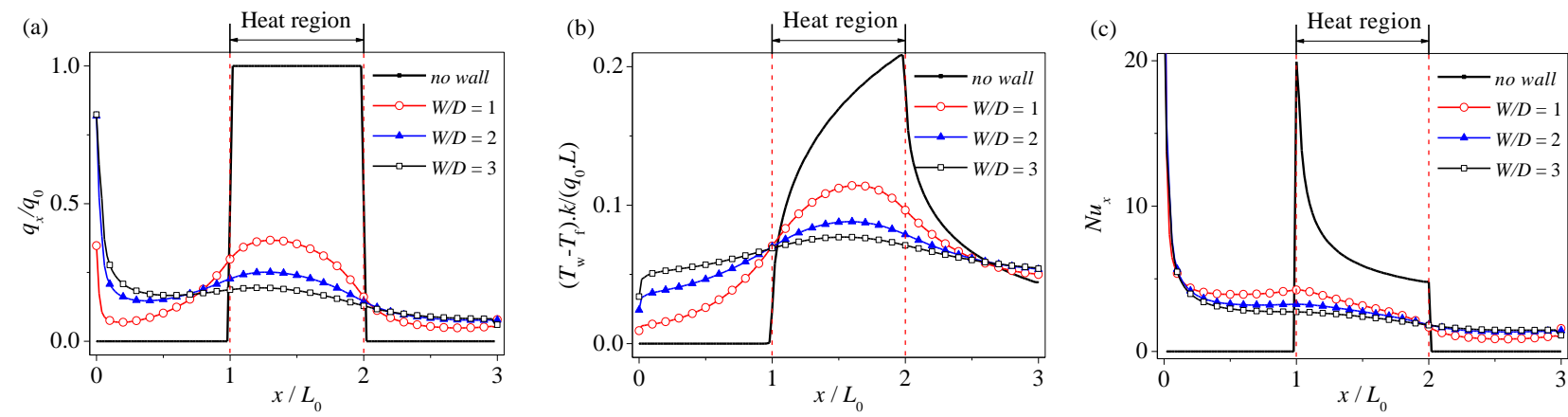

Fig. 9 Effects of $W / D$ on (a) $q_{x} / q_{0}$, (b) $\left(T_{w}-T_{f}\right) * k_{f} /\left(q_{0} * L_{0}\right)$ and (c) $N u_{x}$ for $k_{w} / k_{f}=75, P e=100$.
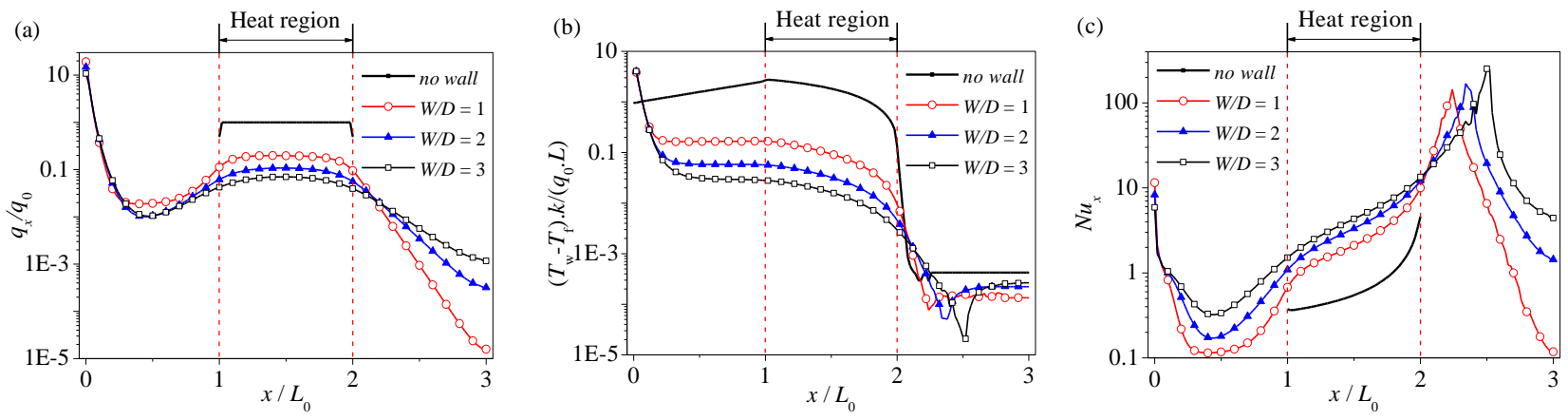

Fig. 10 Effects of $W / D$ on (a) $q_{x} / q_{0}$, (b) $\left(T_{w}-T_{f}\right) * k_{f} /\left(q_{0} * L_{0}\right)$ and (c) $N u_{x}$ for $k_{w} / k_{f}=3, P e=0.1$.

At $k_{w} / k_{f}=75$, affected by the wall conduction, a peak of $N u_{x}$ appears near channel entrance (Fig. 9(c)). The small difference of $N u_{x}$ near channel entrance $\left(x / L_{0}=0\right)$ indicates that $W / D$ has little influence on convection near channel entrance for high $P e$. Comparison of the $N u_{x}$ in Fig. 7 and Fig. 9 indicates that the increase of $W / D$ has an effect on $N u_{x}$ equivalents to increasing the conductivity of the wall. Thermal resistance of the conduction is inversely proportional to the wall thickness, so the thermal resistance of conduction decreases with increase of $W / D$, this cause more heat to move axially, and that leads the decrease of $N u_{x}$, and this trend is in conform with the conclusions of Kosar (2010)..

Different from the results shown in Fig. 9, Fig. 10 demonstrates the curves for $P e=0.1$ when $k_{w} / k_{f}=3$. Compared the curves in Fig. 8 with those in Fig. 10, one can conclude that the effects which caused by the increase of $W / D$ is similar to the effects owing to the increase of $k_{w} / k_{f}$.

\subsection{The Analysis of the Mean Nusselt Number on Heat Region}

Peclet number describes the ratio of convection and conduction in the fluid. For $P e=0.1$, the convection in heat transfer is relatively weak. With the increase of $k_{w} / k_{f}$, the proportion of the heat transferred by conduction of the wall 
increases, and the heating effect of the wall for fluid is strengthened, which leads to the increase of the $\overline{N u}$. From Fig. 11(a), at $P e=0.1$, we can conclude that $\overline{N u}$ is proportional to the wall thermal conductivity for $k_{w} / k_{f} \leq 30$, while $\overline{N u}$ approximately keep constant when $k_{w} / k_{f} \geq 30$.

In order to study the effect of the wall thermal conductivity on $\overline{\mathrm{Nu}}$ at high $\mathrm{Pe}$ conditions, the distribution of the $\overline{N u}$ along with $k_{w} / k_{f}$ when $P e=100$ is also plotted in Fig. 11(a). For the higher convective heat transfer capability at high $P e$ condition $(P e=100)$, the $\overline{N u}$ of the heat region shows a quicker increase than the $\overline{N u}$ at low $P e$ condition $(P e=$ $0.1)$.

To analyze the effect of $P e$ on the distribution of $\overline{\mathrm{Nu}}$ on heat region, Fig. 11(b) gave the distribution of $\overline{\mathrm{Nu}}$ at different $P e$ conditions. As shown in Fig. 11(b), $\overline{N u}$ on heat region rises with the increase of $P e$. A slight increase of the $\overline{N u}$ on heat region is observed as the increase of $P e$ when $P e \leq 1$ since the heat is mainly transferred with diffusion. When $P e$ $>10$, the convection dominants the heat transfer between the heated wall and fluid. Therefore, an obvious increase of $\overline{N u}$ is shown in Fig. 11(b) with the increase of $P e$. For high $P e$ flow, the effect of the wall conduction is not significant, and the heat transfer enhancement becomes weak along with the increase of $P e$. As shown in Fig. 11(b), when $P e>50$, the increase tread of $P e$ is not obvious, and $P e$ almost keeps constant.

(a)

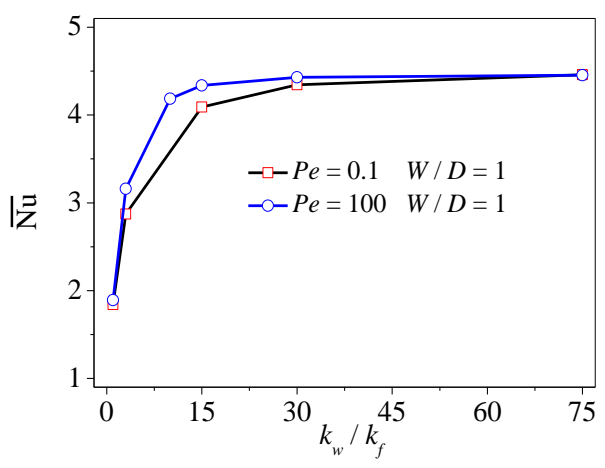

(b)

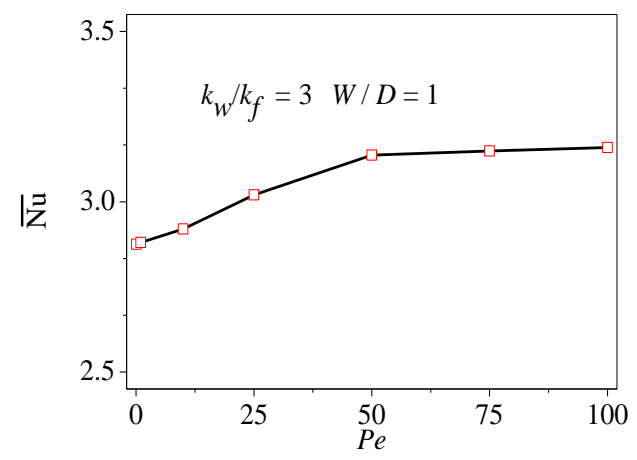

Fig. 11 Effects of $k_{w} / k_{f}$ and $P e$ on $\overline{N u}$ of heat region $\left(1<x / L_{0}<2\right)$.

\section{Conclusions}

Three-dimensional fluid flow and conjugate heat transfer in heat sinks with rectangle microchannels have been analyzed numerically. The continuum model, which includes the conventional Navier-Stokes equations and energy equation are used. The validation of this approach has been demonstrated by comparisons with analytical solutions. Results were obtained for the detailed description of the local and average heat transfer characteristics including wall and fluid temperatures, heat flux, Nusselt number. Effects of the Peclet number $(\mathrm{Pe})$, the relative conductivity $\left(k_{w} / k_{f}\right)$ and the relative thickness $(W / D)$ of solid substrate were presented and discussed. The following conclusions can be drawn from the present results:

1) At low $P e$ flow, axial conduction dominates the heat transfer, which results in a large upstream region which is pre-warmed by axial conduction, so axial conduction cannot be neglected at small $P e$. The increase of $P e$ induces an enhancement of convection, the heat exchange mainly happens on heat region by convection under this condition. The increases of $q_{x} / q_{0}$ on heat region also imply the enhancement of convection when $P e$ rises. Although the temperatures of wall and fluid fall, the temperature difference between wall and fluid becomes more pronounced as $P e$ rises.

2) A more uniform streamwise temperature distribution at fluid-solid interface indicates the enhancement of the pre-warmed effect along with the increase of $k_{w} / k_{f}$. Affected by the entrance region, a peak of $N u_{x}$ has been found near channel inlet for different $P e$. Also, a local peak of $N u_{x}$ appears near entrance of heat region for low conductivity material. The study also reveals that the $N u_{x}$ of low $k_{w} / k_{f}$ material is higher than the $N u_{x}$ of high $k_{w} / k_{f}$ material.

3) The increase of wall thickness has an effect on axial distribution of $N u_{x}$, the trend is similar to that of an increase of $k_{w} / k_{f}$.

4) Compared with the average Nusselt number of difference $P e$ and different $W / D$, it was concluded that at condition of $P e=0.1, \overline{N u}$ is proportional to the wall thermal conductivity for $k_{w} / k_{f} \leq 30$, while $\overline{N u}$ approximately keep constant when $k_{w} / k_{f} \geq 30$. At high $P e$ condition $(P e=100)$, the convection between the wall and fluid is the dominate 
factor in heat transfer, so $\overline{N u}$ of the heat region shows a more quickly increase than the $\overline{N u}$ at low $P e$ condition $(P e=$ 0.1 ). $\overline{N u}$ on heat region rises with the increase of $P e$. The fluid is heated by the wall by means of convection on heat region, when $P e \leq 1$, a slight increase of the $\overline{N u}$ on heat region is shown as the increase of $P e$. When $P e>10$, the convection is the dominant factor in heat transfer, an obvious increase of $\overline{N u}$ is shown with increase of $P e$. For high $P e$ flow, the heat transfer enhancement as increase of $P e$ is weak.

\section{Acknowledgments}

This work was supported by National Natural Science Foundation of China (Grant Nos. U1737104 and 51776037), the Natural Science Foundation of Jiangsu Province (Grant No. BK20170082), and the Special Program for Applied Research on Super Computation of the NSFC-Guangdong Joint Fund (the second phase).

\section{References}

Ashraf M. J., Ali H. M., Usman H., Arshad A., Experimental passive electronics cooling: Parametric investigation of pin-fin geometries and efficient phase change materials, International Journal of Heat and Mass Transfer, Vol. 115 (2017), pp. 251-263.

Ates, A., Darıc1, S., Bilir, S., Unsteady conjugated heat transfer in thick walled pipes involving two-dimensional wall and axial fluid conduction with uniform heat flux boundary condition, International Journal of Heat and Mass Transfer, Vol. 53 (2010), pp. 5058-5064.

Cetin, B., Yazicioglu, A. G., Kakac, S., Slip-flow heat transfer in microtubes with axial conduction and viscous dissipation-An extended Graetz problem, Int. J. Thermal Sci., Vol. 48 (2009), pp. 1673-1678.

Chen, Y. P., Zhang, C. B., Shi, M. H. and Wu, J. F., Three-dimensional numerical simulation of heat and fluid flow in noncircular microchannel heat sinks, Int. Commun. Heat Mass Transfer., Vol. 36 (2009), pp. 917-920.

Chen Y., Yao F., Shi M., Thermal response of a heat pipe with axially " $\Omega$ "-shaped microgrooves, International Journal of Heat and Mass Transfer, Vol. 55 (2012), pp. 4476-4484.

Cole, K. D., Cetin, B, The Effect of Axial Conduction on Heat Transfer in a Liquid Microchannel Flow, Int. J. Heat Mass Transfer, Vol. 54 (2011), pp. 2542-2549.

Deng Z., Zheng Y., Liu X., Zhu B. and Chen Y., Experimental study on thermal performance of an anti-gravity pulsating heat pipe and its application on heat recovery utilization. Applied Thermal Engineering, Vol. 125 (2017), pp. 1368-1378.

Guo, Z. Y., Li, Y., Wang, B. X., A novel concept for convective heat transfer enhancement, Int. J. Heat Mass Transfer, Vol. 41 (1998), pp. 2221-2225.

Guo, Z. Y., Li, Z. X., Size Effect on single-phase channel flow and heat transfer at microscale, Int. J. Heat Fluid Flow, Vol. 24 (2003), pp. 284-298.

Herwig, H., Hausner, O., Critical view on "new results in micro-fluid mechanics": an example, Int. J. Heat Mass Transfer, Vol. 46 (2003), pp. 935-937.

Hetsroni, G., Mosyak, A., Pogrebnyak, E. and Yarin, L. P., Heat transfer in micro-channels: Comparison of experiments with theory and numerical results, Int. J. Heat Mass Transfer, Vol. 48 (2005), pp. 5580-5601.

Jeong, H. E., Jeong, J. T., Extended Graetz problem including streamwise conduction and viscous dissipation in microchannels, Int. J. Heat Mass Transfer, Vol. 49 (2006), pp. 2151-2157.

Kosar, A., Effect of substrate thickness and material on heat transfer in microchannel heat sinks, Int. J. Thermal Sci., Vol. 49 (2010), pp. 635-642.

Li, Z., He, Y. L., Tang, G. H. and Tao, W. Q., Experimental and numerical studies of liquid flow and heat transfer in microtubes," Int. J. Heat Mass Transfer, Vol. 50 (2007), pp. 3447-3460.

Liu, H. L., Shao, X. D., Jia, J. Y., Effects of axial heat conduction and viscous dissipation on heat transfer in circular micro-channels, Int. J. Thermal Sci., Vol. 66 (2013), pp. 34-41.

Liu X., Chen Y., Transient thermal performance analysis of micro heat pipes, Applied Thermal Engineering, Vol. 58 (2013), pp. 585-593.

Liu X., Chen Y., Shi M., Dynamic performance analysis on start-up of closed-loop pulsating heat pipes (CLPHPs), International Journal of Thermal Science, Vol. 65 (2013), pp. 224-233.

Liu X., Chen Y., Fluid flow and heat transfer in flat-plate oscillating heat pipe, Energy and Buildings, Vol. 75 (2014), pp. 29-42. 
Mudawar, I., Assessment of High-Heat-Flux Thermal Management Schemes, IEEE Trans. Compon. Packag. Technol., Vol. 24, no. 2 (2001), pp. 122-141.

Moharana, M. K., Agarwal, G., Khandekar, S., Axial conduction in single-phase simultaneously developing flow in a rectangular mini-channel array, Int. J. Thermal Sci., Vol. 50 (2011), pp. 1001-1012.

Maranzana, G., Perry, I., Maillet, D., Mini- and micro-channels: influence of axial conduction in the walls, Int. J. Heat Mass Transfer, Vol. 47 (2004), pp. 3993-4004.

Nonino, C., Savino, S., Del Giudice, S. and Mansutti, L., Conjugate forced convection and heat conduction in circular microchannels, Int. J. Heat Fluid Flow, Vol. 30 (2009), pp. 823-830.

Qu, W. L., Mudawar, I., Analysis of three-dimensional heat transfer in micro-channel heat sinks, Int. J. Heat Mass Transfer, Vol. 45 (2002), pp. 3973-3985.

Rahimi, M., Mehryar, R., Numerical study of axial heat conduction effects on the local Nusselt number at the entrance and ending regions of a circular microchannel, Int. J. Thermal Sci., Vol. 59 (2012), pp, 87-94.

Shah, R. K., London, A. L., Laminar flow forced convection in ducts: a source book for compact heat exchanger analytical data, Supl. 1 (1978), Academic Press, New York.

Tuckerman, D. B., Pease, R. F. W., High-performance heat sinking for VLSI, IEEE Electron Device Lett., Vol. EDL-2, No.5 (1981), pp.126-129.

Wang, J. R., Min, J. C., Song, Y. Z., Field coordination analysis of convective heat transfer in thermal entrance region in rectangular ducts with one side heated, J. ENG. THERMOPHYS, Vol. 25, no.3 (2004), pp. 478-480.

Zhang, S. X., He, Y. L., Lauriat, G. and Tao, W. Q., Numerical studies of simultaneously developing laminar flow and heat transfer in microtubes with thick wall and constant outside wall temperature, Int. J. Heat Mass Transfer, Vol. 53 (2010), pp. 3977-3989. 